\title{
Unspecified Premix Dosage Form Category
}

National Cancer Institute

\section{Source}

National Cancer Institute. Unspecified Premix Dosage Form Category. NCI Thesaurus.

Code C149863.

A type of specialized pharmaceutical dose form that is used in the preparation of products such as medicated feeding stuffs. 